\title{
Diagnóstico de indicadores de violencia escolar en adolescentes
}

\section{Diagnosis of indicators of school violence in adolescents}

\author{
PACHECO-AMIGO, Beatriz Mabel $\uparrow *$ \\ Universidad Autónoma de Zacatecas, México \\ ID $1^{\text {er }}$ Autor: Beatriz Mabel, Pacheco-Amigo / ORC ID: 0000-0002-8053-3506, Researcher ID Thomson: P-7758-2016, \\ CVU CONACYT ID: 5147251
}

\begin{abstract}
Resumen
El adolescente manifiesta un conjunto de intereses que conforman, mediante la violencia escolar, conflictos conductuales y emocionales en el transcurso del desarrollo y ajuste social, por ello, la descripción y medición de los principales tipos de violencia proyecta un peligro en áreas escolares, especialmente por la normalización de dicha agresividad. Objetivo: diagnosticar el tipo de conducta agresiva del adolescente de edades de 12 a 15 años, mediante pruebas de Tamizaje, integrando así grupos focales dentro de un marco institucionalizado educativo, mediante un estudio de tipo cuantitativo de corte descriptivotransversal, no experimental. Metodología: Para lograr el objetivo se utilizaron diversas técnicas de evaluación la primera fue Resolución y perspectiva de conflicto de violencia escolar, seguido del Instrumento de Acoso y Violencia Escolar (AVE) mismas que fundamentaron la fase de diagnóstico en una muestra analizada de $\mathrm{n}=568$ alumnos. Contribución: se pudo identificar el alto nivel de negligencia de crianza, como también el deterioro de autoconcepto, ideas suicidas y falta de apego a figuras parentales.
\end{abstract}

Violencia escolar, Diagnóstico, Factores de riesgo

\begin{abstract}
The adolescent manifests a set of interests that, through school violence, make up behavioral and emotional conflicts in the course of development and social adjustment, therefore, the description and measurement of the main types of violence projects a danger in school areas, especially due to the normalization of such aggressiveness. Objective: to diagnose the type of aggressive behavior of adolescents aged 12 to 15 years, through screening tests, thus integrating focus groups within an institutionalized educational framework, through a study quantitative type of descriptive-transversal cut, not experimental. Methodology: To achieve the objective, various evaluation techniques were used, the first was Resolution and perspective of school violence conflict, followed by the Instrument for School Harassment and Violence (AVE), which based the diagnosis phase in an analyzed sample of $n=568$ students. Contribution: the high level of parenting neglect could be identified, as well as the deterioration of self-concept, suicidal ideas and lack of attachment to parental figures.
\end{abstract}

\section{School violence, Diagnosis, Risk factors}

Citación: PACHECO-AMIGO, Beatriz Mabel. Diagnóstico de indicadores de violencia escolar en adolescentes. Revista Ciencias de la Educación. 2020. 4-11: 1-8

\footnotetext{
* Correspondencia del Autor (correo electrónico: bpachecoamigo@yahoo.com.mx)

$\dagger$ Investigador contribuyendo como primer autor.
} 


\section{Introducción}

La violencia escolar, como lo refiere la Organización Mundial de la Salud (2003), cubre una amplia gama de consecuencias como los daños psíquicos incluyendo las privaciones y las deficiencias del desarrollo. Por ello, cobra relevancia los actos de violencia que no provocan lesiones y cuyas consecuencias pueden ser inmediatas o latentes, atendiendo el efecto global de las personas en las comunidades y en la sociedad en general (p. 13).

La importancia de ser atendida la violencia escolar dentro del contexto educativo, se presenta por diversos factores, incluidos aquellos de riesgo, que se relacionan implícitamente con vínculos amistosos entre pares, que buscan la integración a grupos sociales que le permiten al menor articular intereses y gustos en común que ayudan a fusionar ideas (Olweus, 1993), aún cuando no sean las apropiadas dentro del desarrollo.

La violencia escolar en México ha sido un fenómeno social que se ha incrementado en la última década, Miglino (2019) señala en "La Investigación de la Organización No Gubernamental (ONG) Internacional Bullying Sin Fronteras para América Latina y España, realizado entre abril de 2017 y abril de 2018, los problemas de violencia escolar en México van en crecimiento, donde 7 de cada 10 adolescentes soportan todos los días algún de tipo de violencia escolar". (p.4)

Por otra parte, (referido por el mismo autor) investigaciones realizadas con apoyo de la Organización No Gubernamental (ONG) Internacional Bullying Sin Fronteras y la Organización para la Cooperación y el Desarrollo Económico(OCDE), ubican a México como país con severos casos de bullying escolar, con una población que alcanza los 40 millones de estudiantes de nivel primaria y secundaria en México, originando resultados alarmantes de presencia progresiva y sistemática de algún tipo de violencia que toleran alrededor de 28 millones de niños y adolescentes.
Desde un contexto global, la correspondencia de violencia escolar sitúa a México en primer lugar a nivel mundial, seguido por los Estados Unidos de América, China, España, Japón, Guatemala, República Dominicana, Costa Rica, Honduras, El Salvador, Brasil, Argentina, Chile, Uruguay, Bélgica, Italia, Suecia, Francia, Dinamarca y Noruega; situación que enmarca el Primer Estudio Mundial referido por la Organización No Gubernamental con apoyo de la Organización para la Cooperación y el Desarrollo Económico (OCDE) para América, Europa, África, Oceanía y Asia; realizado entre junio de 2017 y junio de 2018, mismos resultados que se encontraron que los casos de Bullying en todo el mundo han ido en aumento de manera inusitada con relación a las últimas mediciones (Miglino, 2019).

Los temas de violencia escolar, referido por Pacheco (2014), hace imperante la necesidad de conformar planes de intervención, con estrategias que afronten el fenómeno social, por ello, las nociones del cambio comportamental se ejerce desde diversas posturas asociadas al fenónemo, una de ellas es la triada entre agresor, víctima y espectadores. Además de aunar la incapacidad de sectores institucionales para brindar respuestas en contextos sociales inmediatos, donde los jóvenes han construido sentido de pertenencia en base a la barrera de grupos de pares que favorece el pobre control de emociones mediante expresiones de agresividad (Kornblit, 2008).

De esta manera, el problema de estudio, la violencia escolar en alumnos de secundaria, se describieron factores de riesgo en el ámbito familiar, social y académico del menor. Asimismo, para brindar soporte teórico fue preciso establecer modelos desde una perspectiva integral que contribuyen a la violencia escolar, permitiendo anclar factores de riesgo e indicadores de vulnerabilidad afectivo/social del menor (Morcote, Rodríguez \& Enamorado, 2012).

La presente investigación, como estudio de la conducta de menores, relacionado al diagnóstico de la violencia escolar, refiere consecuencias en el autoconcepto, ideaciones específicas y justificación social de la violencia como medio para lograr objetivos específicos (Smith, 2000). 


\section{Adolescencia}

La adolescencia está determinada por un conjunto de cambios estructurales donde se encuentran los sociales, psicológicos y fisiológicos, que permiten al menor conformar parte de su identidad transitoria hasta llegar a la conformación de la personalidad; por este motivo, se define como una etapa de innovación dentro de su paso de progreso y proceso (González, 2008).

El proceso de la adolescencia se forja como un ambiente afectivo donde el sujeto se encuentra en una etapa de locomoción social, por ello, los campos sociales y psicológicos en los que se desenvuelve no están aún estructurados de forma clara, en virtud de que no tiene un enfoque relevante para la comprensión de las obligaciones sociales ni del estatus que ellos refieren, escenario que se ve expresado en el comportamiento como resultado de esta indecisión.

En consecuencia, la adolescencia, vista como una fase de proceso y crecimiento del individuo, comprende no solo cambios de orden físico sino también constituye múltiples formas de cambio de orden social, expresado en términos psicológicos, se denomina como una etapa progresiva que comprende entre los $12 \mathrm{y}$ 18 años, es decir, la adolescencia se localiza entre la edad de la pubertad y la edad adulta, definida como una etapa de evolución en el que el individuo pasa física y psicológicamente del estado de ser un niño a la adultez, según Hurlock (2014). Por ello, la Organización Mundial de la Salud (2018) hace referencia a conductas de alto riesgo por considerarse una etapa de vulnerabilidad dentro del desarrollo determinado biológicamente.

Desde la perspectiva moral, el desarrollo integral de los adolescentes es un estado de proceso de maduración, el estudiante logra y edifica en sus procesos de acción comportamientos que se manejan por principios morales y éticos de carácter universal, de esta forma, en la violencia escolar, el nivel de agresión y el rol del agresor, en contextos educacionales, se establece un bajo nivel de principios morales que conserva la persona.
Por lo mismo, adquiere características que le permite tener conductas de acoso, agresión, daño físico, psicológico y moral a otro estudiante, con las características que son propias de la violencia escolar (Kohlberg, 1992), aún, cuando el adolescente pretenda lograr una armonía aparente; el nivel de adaptación en relación al ambiente impulsa la descompensación en el equilibrio psíquico, necesario en el proceso social del individuo, mencionado en Pacheco, Lozano, González (2018).

\section{Consecuencias del desarrollo inadecuado del menor}

El paso significativo en el desarrollo que realiza el niño hacia la adolescencia, exige un avance de socialización que permita establecer vínculos complejos ausentado de los padres, por ello, la complejidad del joven relacionado a su conducta y a sus pensamientos pueden ser origen de conductas inestables el que provoca descontrol individual y familiar, por ser un proceso de ajuste y pseudoestabilizador de la fase evolutiva y del desarrollo del menor (Aberastury, 2014).

Dentro de los problemas que presenta el adolescente, uno de ellos, es que, al comenzar la adolescencia se formula una nueva creación del rol social, lo que ocasiona una serie de contradicciones y confusión de orden social obstaculizando los escenarios educativos, de esta forma, la violencia escolar está determinada por la recreación de un período de largo tiempo, que por lo regular es consumada por un alumno pero apoyado por un grupo ya sea de espectador o neutral (Smith, 2000), tomando como característica una familiaridad del vínculo que produce la violencia escolar entre los mismos grupos de pares (Ortega, 2006).

Desde el punto de vista psicológico, los adolescentes que presentan conductas disruptivas pueden presentar con anticipación una serie de conductas que formalizan conductas inadaptadas dentro del desarrollo, según Aberastury (2014), refiere una serie de indicadores comportamentales que coadyuvan a generar una adolescencia psicopática donde se identifican las siguientes características (Ver tabla 1): 
Conductas psicopáticas del adolescente

Necesita estar con otras personas para dar aval a la estima. Tiene un insight defensivo y lo utiliza para su manejo.

La forma de comunicación es mediante la acción. La experiencia no sirve para adquirir aprendizaje.

Niega el afecto y sentimientos de pérdida.

No tiene adapatación social por no alcanzar una identidad plena.

Tabla 1 Tipos de conductas

Por otra parte, se establece patrones específicos de violencia dentro del aula y el entorno escolar, es decir, presenta diversas formas de manifestación conductual, que dependerán del tipo de vínculo que se organice entre la víctima y el agresor, generando la clasificación de violencias explícitas las que destacan según Banco Mundial (2011) (Ver tabla 2):

\begin{tabular}{|c|c|}
\hline Tipo de violencia & Características \\
\hline $\begin{array}{ll}\text { Violencia } & \text { Física } \\
\text { directa } & \end{array}$ & $\begin{array}{l}\text { Está catalogada por dar patadas, } \\
\text { codazos, golpes con las manos, escupir, } \\
\text { morder de manera física al individuo, } \\
\text { eventos vergonzosos como bajar los } \\
\text { pantalones, jalar la ropa y tirarla a los } \\
\text { botes de basura, entre otros, por } \\
\text { mencionar algunas caracterizas de este } \\
\text { tipo de violencia. }\end{array}$ \\
\hline $\begin{array}{l}\text { Violencia } \quad \text { Física } \\
\text { indirecta }\end{array}$ & $\begin{array}{l}\text { Son actos que causan perjuicio o } \\
\text { quebranto en las posesiones de las y los } \\
\text { alumnos como la sustracción, } \\
\text { destrucción, pérdida, o detención de } \\
\text { objetos u otras posesiones. }\end{array}$ \\
\hline $\begin{array}{l}\text { Violencia } \\
\text { Psicológica }\end{array}$ & $\begin{array}{l}\text { El medio que utilizado son chantajes, } \\
\text { ofensas, menosprecio integrando el uso } \\
\text { de espacios virtuales por medio de las } \\
\text { nuevas tecnologías como chats, blogs, } \\
\text { redes sociales, correo electrónico, } \\
\text { mensajes de texto, servidores que } \\
\text { almacenan videos o fotografías, páginas } \\
\text { web, entre otros (ciberbullying). }\end{array}$ \\
\hline Violencia Sexual & $\begin{array}{l}\text { Identificada como toda acción o } \\
\text { negligencia que incita y sitúa en peligro } \\
\text { o lastima la autonomía, seguridad e } \\
\text { integridad y progreso psicosexual de las } \\
\text { y los estudiantes, como miradas o frases } \\
\text { impúdicas, persecución, prácticas } \\
\text { sexuales no voluntarias, acoso, violación } \\
\text { o el uso vergonzoso de la imagen de las } \\
\text { y los alumnos a través de las tecnologías } \\
\text { de la información y comunicación. }\end{array}$ \\
\hline Violencia Verbal & $\begin{array}{l}\text { Identificada como aquellos sucesos } \\
\text { violentos que se ejercen en el uso de la } \\
\text { palabra, como insultos, poner alias, } \\
\text { someter, repudiar en público, ironías } \\
\text { sobre la vestimenta, aspecto físico, raza y } \\
\text { origen étnico. }\end{array}$ \\
\hline Violencia Social & $\begin{array}{l}\text { Identificada como la conducta de soledad } \\
\text { incitada por el provocador hacia la } \\
\text { víctima. Se puede mostrar no } \\
\text { dirigiéndole la palabra al adolescente } \\
\text { acosado, negándole el saludo, } \\
\text { incomunicándolo o creando chismes que } \\
\text { perturben su persona. }\end{array}$ \\
\hline
\end{tabular}

Tabla 2 Análisis de violencia según Banco Mundial Fuente: Extraído de Pacheco (2019)

\section{Causas y factores individuales de riesgo en la violencia escolar}

Al evaluar las causas y factores de riesgo de la violencia escolar, es imprescindible establecer, que suelen originarse por diversos criterios del sujeto agredido, como el tener características de baja autoestima o pobre conceptualización de ellos mismos, presentar algún tipo de discapacidad, rasgos físicos o culturales diferentes, estar dentro de las minorías étnicas y/o raciales - culturales; desde la perspectiva conductual el mantener un pobre control de conductas que se evalúa mediante un comportamiento agresivo o desafiante, constante impulsividad, trastornos afectivos (depresión y distimia), trastorno por déficit de atención con hiperactividad complementándose con el cuadro de violencia escolar por mantener una falta de actitud/aptitud para resolver problemas, constantes conductas de riesgo como lo son el uso y abuso de alcohol, drogas y pandillerismo, Pacheco ( 2019).

Asimismo, como causas de riesgo en la violencia escolar se presentan en diversas áreas específicas, como lo señala el Banco Mundial (2011), se encuentran los factores y agentes patognomónico, quienes serán los que ayuden a establecer estados de alarma dentro de una institución educativa, en consecuencia de la interacción e interrelación de factores individuales que se articulan y potencian con los factores sociales y áreas interpersonales del menor para generar conductas inapropiadas dentro del contexto escolar. (Rigby, 2004).

\section{Causas de riesgos en la violencia escolar}

De acuerdo con el informe del Banco Mundial (2011), existen varios contextos y áreas que promueven conductas agresivas dentro del contexto escolar en condiciones particulares, como lo son las interpersonales y/o familiares que promueven la reproducción de violencia en una institución escolar que se consideran como factor de riesgo inmediato al integrarse varios de ellos, como lo son:

Falla de contenidos en los padres para desarrollar una paternidad efectiva. Padecimientos mentales en la familia.

- Intervención de la familia en movimientos criminales.

Adicciones en padres de familia o algún miembro de ésta. 
- $\quad$ Aglomeración de las familias.

- Modo opresor/violento ejercido por los padres.

- Violencia familiar.

- Separación familiar.

- Relaciones con pares que sean delincuentes o jóvenes en problema con la autoridad.

Por este motivo, los recursos de las capacidades, situaciones o circunstancias individuales, interpersonales o familiares, escolares, sociales o comunitarias son indispensables en el cuidado y prevención de la violencia en el entorno escolar (Banco Mundial 2011), donde surgen los factores protectores del sujeto, que brindarán la posibilidad de tener repertorio conductual vinculado al comportamiento prosocial del adolescente designados en cuatro áreas (Ver tabla 3):

\begin{tabular}{|c|c|}
\hline $\begin{array}{l}\text { Ambiente } \\
\text { individual }\end{array}$ & 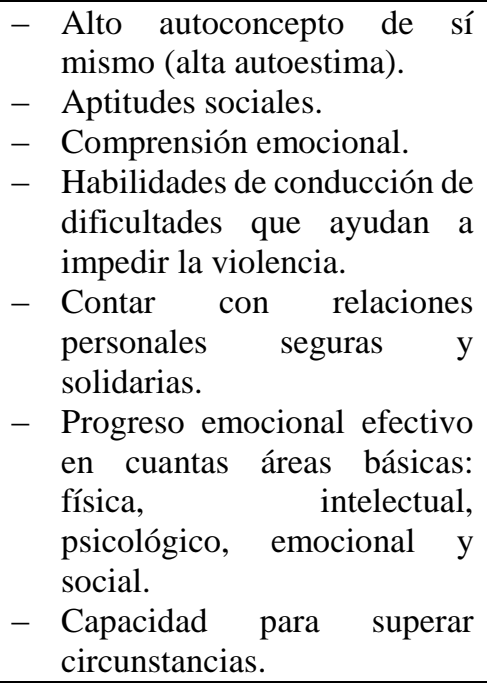 \\
\hline $\begin{array}{l}\text { Ambiente } \\
\text { interpersonal } \\
\text { familiar }\end{array}$ & $\begin{array}{ll}\text { - } & \text { Vigilancia por parte de los } \\
\text { padres de familia. } \\
- & \text { Paternidad objetiva. } \\
- & \text { Fortalecimiento y progreso de } \\
\text { destrezas personales y } \\
\text { sociales en los hijos. }\end{array}$ \\
\hline Espacio escolar & $\begin{array}{ll}- & \text { Conducta y reglas claras de } \\
& \text { comportamiento. } \\
- & \text { Buena calidad de enseñanza. } \\
- & \text { Ambiente } \\
& \text { responsable. }\end{array}$ \\
\hline $\begin{array}{l}\text { Ambiente social y } \\
\text { comunitario }\end{array}$ & 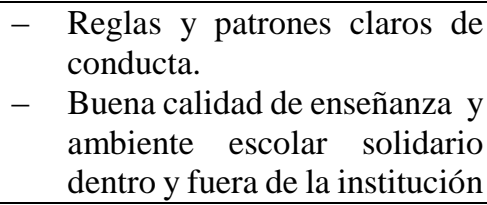 \\
\hline
\end{tabular}

Tabla 3 Factores de protección según el Banco Mundial Fuente: Elaboración Propia

\section{Metodología}

La Metodología utilizada fue mediante grupo focal, de corte cuantitativo de inferencia lineal mediante un diseño no experimental, que permitió tener una perspectiva objetiva del fenómeno de la violencia escolar, con la extracción de datos que apoyaron la investigación permitiendo realizar estimaciones mediante procesos de Tamizaje (Prueba estandarizada a nivel de Gobierno Federal, lo que auxilio a describir indicadores que fundamentan el estudio mediante la selección de muestra).

De esta forma, la muestra del estudio fue de tipo no probabilística de tipo intencionado, de estudiantes de educación Secundaria de la cabecera Municipal de Zacatecas, cuyo criterio de inclusión se estableció en adolescentes de 12 a 15 años, que hubieran presentado algún tipo de violencia escolar o agresor identificado por la institución educativa, menores que se encuentran cursando estudios de secundaria de forma regular y posteriormente el protocolo de consentimiento informado por padres o tutores, quedando la distribución de la siguiente manera: (Ver tabla 4).

\begin{tabular}{|l|r|}
\hline \multicolumn{1}{|c}{ Escuelas } & $\begin{array}{c}\text { Número de } \\
\text { Alumnos }\end{array}$ \\
\hline Secundarias Generales Federales & 237 \\
\hline Secundaria General Estatal & 142 \\
\hline Secundaria General Particular & 51 \\
\hline Secundaria Técnica & 78 \\
\hline Telesecundarias & 60 \\
\hline
\end{tabular}

Tabla 4 Distribución muestral de la población Estudiantil de Zacatecas de Educación Secundaria

Fuente: Elaboración Propia

Las técnicas que se utilizaron fueron técnicas primarias de investigación, como la entrevista semiestructurada (Resolución y perspectiva de conflictos en el tema de violencia escolar) avalado mediante el Departamento de Prevención del Delito de Seguridad Pública del Estado, como también el instrumento de medición de Acoso y Violencia Escolar (AVE) de Piñuel \& Oñate ( 2013), estandarizado en población mexicana con un alfa de Cronbach del 0.95 constituido por 94 ítems, abordando los tipos de violencia que se dan en contexto educativo de secundaria e indicadores de factores de riesgos asociados a la violencia escolar. 


\section{Resultados}

Con la finalidad de poder realizar un diagnóstico de la violencia ejercida entre los menores adolescentes de 12 a 15 años, de la muestra estudiada se pudo identificar lo siguiente (Ver tabla 5):

\begin{tabular}{|l|r|}
\hline \multicolumn{1}{|c|}{ Tipos de violencia } & Porcentaje \\
\hline Violencia verbal & $43 \%$ \\
\hline Violencia directa & $25 \%$ \\
\hline Violencia social & $14 \%$ \\
\hline Violencia indirecta & $10 \%$ \\
\hline Violencia psicológica & $6 \%$ \\
\hline Violencia sexual & $2 \%$ \\
\hline
\end{tabular}

Tabla 5 Resultado por frecuencia lineal integral en tipos de violencia

Fuente: Elaboración Propia

Por otra parte, al articular los rasgos personales de los adolescentes se pudo identificar (Ver tabla 6):

\begin{tabular}{|l|r|}
\hline \multicolumn{1}{|c|}{ Indicadores } & Porcentaje \\
\hline Idea Suicida & $68 \%$ \\
\hline Pobre concepto de sí mismo & $73 \%$ \\
\hline Rasgos depresivos & $52 \%$ \\
\hline Responde con agresión & $34 \%$ \\
\hline Problemas con autoridad escolar & $62 \%$ \\
\hline Participación pasiva de violencia escolar & $94 \%$ \\
\hline Sensación de desvalimiento & $87 \%$ \\
\hline Percepción de negligencia parental & $91 \%$ \\
\hline Factor de riesgo interpersonal o familiar & $82 \%$ \\
\hline
\end{tabular}

Tabla 6 Resultados por frecuencia lineal general de rasgos personales de la muestra

Fuente: Elaboración Propia

\section{Hallazgos articulados a indicadores de violencia escolar en adolescentes}

Con los datos expuestos en las tablas de resultados, relacionado a los instrumentos aplicados, se puede establecer que los datos integrales hacen referencia que el $43 \%$ de los adolescentes tienen mayor incidencia en la violencia verbal, integrando el porcentaje mínimo del $2 \%$ en la violencia sexual.

Por otra parte, con los datos expuestos en forma de frecuencia simple es importante señalar que el $94 \%$ de los adolescentes evaluados, han participado en violencia escolar pasiva, seguido del $91 \%$ de la percepción de negligencia de las figuras parentales. De esta misma manera, la importancia que abarca el $87 \%$ de la muestra de mantener una sensación de desvalimiento ante la violencia escolar y un $82 \%$ de factores de riesgo interpersonal o familiar.
En la evaluación, es importante destacar los datos sobresalientes de funciones de auto percepción negativas por parte del adolescente que alcanza el $73 \%$ de la muestra y un $68 \%$ de menores que presentan ideas suicidas, manteniendo un alto porcentaje problemas con la autoridad y rasgos depresivos.

\section{Agradecimientos}

Agradecimiento a la Secretaría de Educación Pública del Estado de Zacatecas; Proyecto avalado por el Centro de Investigación Psicológico de la Universidad Autónoma de Zacatecas, México.

\section{Conclusiones}

El propósito de la investigación fue inicialmente identificar la población con problemas de violencia escolar, por lo que se realizó en primer instancia un proceso de Tamizaje, de esta manera, al ser identificados se pudieron integrar y aplicar los instrumentos específicos para describir indicadores relacionados a tipos de violencia y rasgos personales de la muestra. Datos, que permitieron establecer una descripción de violencia y rasgos personales de los menores que se articulan con los referentes teóricos expuestos.

Por otra parte, la violencia escolar, en la muestra estudiada, se infiere los porcentajes obtenidos mediante frecuencias lineales, que se presenta por un incremento de factores de riesgos y a la ausencia de factores de protección, específicamente, el riesgo del factor familiar que aumenta conflictos en el campo psicológico y emocional de los menores, abriendo brechas hacia un factor de riesgo social mayor al mantener un $94 \%$ de violencia pasiva; de esta forma, las probabilidades de falta de apego a figuras parentales positivas refieren el $91 \%$,característica que pone aún más en estado de vulnerabilidad del menor por su fase de desarrollo.

Los datos exhibidos permitió afirmar que existen consistencias en los instrumentos aplicados, en virtud, de que las frecuencias asociadas a tipos de violencia escolar y rasgos personales de la muestra exhiben indicadores de consistencias interna de los instrumentos relacionados entre ellos en la fase descriptiva. 
Relacionados con la gravedad del tipo de violencia y los rasgos personales de los menores, se orienta a una mayor incidencia a la violencia verbal y la directa, pero en relación a los segundos (rasgos personales) se infiere serios problemas de orden conductual, de seguridad pública y de áreas de asistencia de la salud, relacionados a conductas problemáticas con la autoridad y serias alternaciones en el curso del pensamiento como lo son las ideas suicidas, sensación de desvalimiento y una pobre conceptualización de ellos mismos, alcanzando todas éstas sobre el $50 \%$.

En relación a la muestra y datos obtenidos, no existieron datos relevante en relación al género, por lo que se propone aplicar programas psicoeducativos de prevención (primaria, secundaria y terciaria), el cual se debe implementar en la población Diana de adolescente evaluados, por otra parte se sugiere estudios con mayor profundidad clínica en el aspecto de salud mental que permita integrar un diagnóstico pertinente y brindar tratamientos oportunos en los casos que se considere pertinente al existir presencias de vectores patognomónicos en los menores, asimismo, para finalizar la investigación, es significativo mencionar que la población evaluada tiene correspondencia con los marcos referenciales estadísticos como los del Centro de Integración juvenil (2018), establecidos en la población de salud mental, por lo que es imperante realizar programas educativos orientados a instituciones escolares, padres de familias y/o tutores para reestablecer conductas preventivas en lo que respecta a salud comunitaria en aulas libres de violencia escolar que implica una sucesión de factores de estudios multidisciplinarios e intervención de tipo holística.

\section{Referencias}

Aberastury, A. \&. (2014). La adolescencia normal. Buenos Aires, Argentina: Paidos.

Banco Mundial. (2011). Prevención de la violencia a través de las escuelas urbanas de América Latina y el Caribe. Guía práctica. Banco Mundial Heart.

Centro de Integración Juvenil. (2018). Intranet. Acoso Escolar y uso de drogas. México: CIJ.

González, J. (2008). Psicopatología de la adolescencia. México: Manual Moderno.
Hurlock, E. (2014). Psicología de la adolescencia. Buenos Aires: Paidos.

Kohlberg, L. (1992). Psicología del desarrollo moral. Bilbao: Desclée de Brower.

Kornblit, A. (2008). Violencia escolar y climas sociales. Buenos Aires, Argentina: Biblos.

Miglino, J. (2019). Bullying. México Estadística 2017-2018. Equipo Internacional. México: México 2017-2018 Equipo Internacional B.S.F.

Morcote González, O. R.-B.-E. (2012). Percepción de estudiantes de secundaria acerca de la violencia escolar en Tunja, Boyacá, Colombia a partir de la Ley 1620 de convivencia escolar. En Panorama jurídico y sociojurídico de los Derechos Humanos, Sociales y Ambientales. Barranquilla: Corporación Educativa Mayor del Desarrollo Simón Bolivar.

Olweus, D. (1993). Bullying at school. What we Know and we con do. Madrid: Morata.

Organización Mundial de la Salud. (2003). Violencia escolar. Prevención educativa, (pág. 13). México.

Organización Mundial de la Salud. (2018). Salud del adolescente. Temas de salud. Región de las Américas: OMS.

Ortega, R. \&. (2006). Violencia escolar y bullying. Barcelona: Información psicológica.

Pacheco, B. (2014). Agresividad escolar e ideas suicidas en escuelas secundaria. Educación y Humanismo, 16(27), 27-38.

Pacheco, B. (2019). Riesgo de la infancia y la adolescencia. México: Colofon .

Pacheco, B., \& Lozano, J. \&. (2018). Diagnosis of the use of social networks: risk factor for the adolscent. RIDE. Rev. Iberoam. Investig. Desarro. Educ., 8(16), 53-62.

Piñuel, I. \&. Oñate, A. (2013). AVE Acoso y violencia escolar. Madrid, España: Tea.

Rigby, K. (2004). Addressing bullying in schools.Theoretical perspectives and their implications. School Psychology International, 25(3), 287-300. 
Ruiz Martínez, A. O., Vázquez Arévalo, R., Mancilla Díaz, J. M., López-Aguilar, X., \& Álvarez Rayón, G. (Agosto de 2010). Funcionamiento familiar en el riesgo y la protección de trastornos del comportamiento alimentario. Univ. Psychol, IX(2), 447-455.

Smith, P. \&. (2000). Bullying in Schools: Lessons from two decades of research. Agressive Behavior, VII(26), 1-9. 